\title{
Shadowing, entropy and minimal subsystems
}

\author{
T. K. Subrahmonian Moothathu • Piotr Oprocha
}

Received: 13 November 2012 / Accepted: 20 April 2013 / Published online: 7 May 2013

(C) The Author(s) 2013. This article is published with open access at Springerlink.com

\begin{abstract}
We consider non-wandering dynamical systems having the shadowing property, mainly in the presence of sensitivity or transitivity, and investigate how closely such systems resemble the shift dynamical system in the richness of various types of minimal subsystems. In our excavation, we do discover regularly recurrent points, sensitive almost 1-1 extensions of odometers, minimal systems with positive topological entropy, etc. We also show that transitive semi-distal systems with shadowing are in fact minimal equicontinuous systems (hence with zero entropy) and, in contrast to systems with shadowing, the entropy points do not have to be densely distributed in transitive systems.
\end{abstract}

Keywords Shadowing property - Pseudo-orbit - Minimal system · Odometer · Sensitive $\cdot$ Transitive $\cdot$ Weak mixing $\cdot$ Distal

Mathematics Subject Classification (1991) Primary 37B05;

Secondary 34D05 $\cdot 37 \mathrm{~B} 10 \cdot 37 \mathrm{C} 50$

Communicated by H. Bruin.

T. K. S. Moothathu

Department of Mathematics and Statistics, University of Hyderabad, Hyderabad 50004 , India

e-mail: tksubru@gmail.com

P. Oprocha $(\varangle)$

AGH University of Science and Technology, Faculty of Applied Mathematics, Al. A. Mickiewicza 30, 30-059 Kraków, Poland

e-mail: oprocha@agh.edu.pl

P. Oprocha

Institute of Mathematics, Polish Academy of Sciences, U1. Śniadeckich 8, 00-956 Warszawa, Poland 


\section{Introduction}

The concept of shadowing of approximate trajectories entered mathematical literature from the very influential and important works of Anosov and Bowen obtained in early 1970s (e.g. [5,14] contain independent proofs of the so-called classical shadowing lemma; see a book by Pilyugin [29] for more historical remarks on the development of this important topic). On one hand, shadowing is a natural property to consider since it ensures that approximate trajectories "mimic" evolution of true orbits. On the other hand they are present in many systems with sufficient expansion of distance, hence there are many natural examples of systems with this property. Most of the work done on shadowing in the last century was related to hyperbolic systems. While hyperbolicity can be expressed in many equivalent ways, the natural topological assumption is shadowing with some kind of expansivity (e.g. see [26]). It is worth mentioning here that shadowing is a $C^{0}$-generic property (i.e. with respect to the topology of uniform convergence) in various cases (e.g. see $[8,21]$ and the references therein). This gives a motivation for further research on non-hyperbolic systems with shadowing, but also generates many problems, since non-expansive systems with shadowing are much harder to study.

It is worth emphasizing that there is known example [9] of a 3-dimensional manifold with a $C^{1}$-diffeomorphism whose neighborhood (in $C^{1}$-topology on the space diffeomorphisms) consists of maps without shadowing. But even in this case of $C^{1}$ diffeomorphisms on smooth manifolds, there are strong connections between shadowing and stability. First, it was proved by Sakai [31] that the $C^{1}$-interior of the set of diffeomorphisms with the shadowing property coincides with the set of structurally stable diffeomorphisms and recently it was proved by Pilyugin and Tikhomirov [30] that structurally stable diffeomorphisms coincide with the class of diffeomorphisms with Lipschitz shadowing property (in this kind of shadowing, there is a Lipschitz constant $L$ bonding together $\delta$ in the definition of pseudo-orbit with the accuracy of shadowing $L \delta$ ).

In this paper we mainly focus on the structure of minimal subsystems in nonwandering systems with shadowing (the reader who is not familiar with the shadowing property is referred to books [6] or [28,29]). This does not limit generality of our considerations, since it is well known that the restriction of a continuous surjection to its non-wandering set gives a non-wandering system with shadowing (e.g. see section $\$ 3.4$ in [6]). One of the reasons for considering non-wandering maps (e.g. instead of systems having the stronger property of transitivity) is that minimal points (and systems) are dense in every non-wandering system with shadowing [25], so it seems natural that knowledge about the structure of these systems can throw light on the possible types of global dynamical behaviors.

Probably the best known examples of minimal systems are periodic orbits and their generalizations: irrational rotations on the tori and odometers (also called adding machines) on the Cantor set. While irrational rotations do not have shadowing, odometers have this property and furthermore they are the only possible examples of infinite minimal systems with shadowing [23]. While there is no complete characterization of minimal systems, some sufficient conditions on the structure of spaces admitting minimal maps are avilable [7]. What is immediately visible is the richness of possible 
types of minimal systems. In the full shift (Smale's horseshoe) there are uncountably many minimal subsystems, representing various types of dynamics (almost 1-1 extensions of odometers, weakly mixing systems with zero or positive entropy, systems chaotic in various senses etc.). Since full shift is an accessible example of a system with shadowing on one hand, and has very rich dynamics on the other hand, it is interesting to explore as to what extent minimal subsystems of full shift can be present in a less standard system with shadowing.

Our starting point (and main motivation) is [25] where many problems on dynamics of non-wandering systems with shadowing were left open. We answer most of the questions from [25]. Some of our main results are summarized as follows.

(i) Non-wandering systems with shadowing have a dense set of regularly recurrent points (Theorem 3.2). If the system is also sensitive, then non-periodic regularly recurrent points, and minimal points that are not regularly recurrent are also dense in the system (Corollay 4.3).

(ii) In a non-wandering sensitive dynamical system with shadowing, subsystems that are sensitive almost 1-1 extensions of odometers as well as minimal subsystems with positive entropy are densely distributed (Theorems 4.2, 5.2).

(iii) Let $(X, f)$ be a non-wandering sensitive system with shadowing, and let $U \subset X$ be a nonempty open set. Then some power of $f$ has a subsystem contained in $U$ such that the full shift is a factor of this subsystem (Theorem 5.2).

\section{Preliminaries}

A set $A \subset \mathbb{N}$ is syndetic if there is $K>0$ such that $[n, n+K] \cap A \neq \emptyset$ for every $n \in \mathbb{N}$. If $A \subset \mathbb{N}$ is a set such that $\mathbb{N} \backslash A$ is not syndetic, then we say that $A$ is thick. If $\mathbb{N} \backslash A$ is finite, then we say that $A$ is cofinite.

If $(X, d)$ is a compact metric space, $x \in X$ and $r>0$, then $B(x, r)$, and $\bar{B}(x, r)$ denote, respectively, the open and closed ball centered at $x$, and with radius $r$. More generally, for $A \subset X$ and $r>0$, we set $B(A, r)=\{x \in X: \operatorname{dist}(x, A)<r\}$ and $\bar{B}(A, r)=\{x \in X: \operatorname{dist}(x, A) \leq r\}$. Closure of a set $D \subset X$ is as usual denoted as $\bar{D}$.

A pair $(X, f)$ is a dynamical system if $(X, d)$ is a compact metric space and $f: X \rightarrow$ $X$ is a continuous map. The orbit of a point $x \in X$ is the set $O(f, x)=\left\{f^{n}(x): n \geq 0\right\}$. The set of limit points of the orbit $O(f, x)$ is called the $\omega$-limit set of $x$, and is denoted by $\omega(f, x)$.

\subsection{Dynamics of points, pairs and sets}

Given $U, V \subset X$ and $x \in X$ we denote $N(x, U)=\left\{n \in \mathbb{N}: f^{n}(x) \in U\right\}$ and $N(U, V)=\left\{n \in \mathbb{N}: f^{n}(U) \cap V \neq \emptyset\right\}$. A point $x \in X$ is: periodic if there is $n>0$ such that $f^{n}(x)=x$; recurrent if $N(x, U) \neq \emptyset$ for every neighborhood $U$ of $x$; regularly recurrent if $k \mathbb{N} \subset N(x, U)$ for some integer $k=k(U)>0$ and every open neighborhood $U$ of $x$; minimal if $N(x, U)$ is syndetic for any open set $U$ containing $x$. Obviously, every periodic point is regularly recurrent, every regularly recurrent 
point is minimal and every minimal point is recurrent (but not vice-versa). Denote by $P(f), R(f), M(f)$ and $R R(f)$, respectively, the set of all periodic, recurrent, minimal and regularly recurrent points of $f$. We say that $x \in X$ is a non-wandering point if $N(U, U) \neq \emptyset$ for every neighborhood $U$ of $x$. The set of all non-wandering points of $f$ is denoted as $\Omega(f)$. Observe that $\Omega(f)$ is closed and $f(\Omega(f)) \subset \Omega(f)$. If $\Omega(f)=X$, the system is said to be non-wandering. It is known that any non-wandering system has a dense set of recurrent points (e.g. see [18, Theorem 1.27]).

We say that $f$ is transitive if for every pair of nonempty open subsets $U$ and $V$ of $X$ there is an $n \geq 0$ such that $f^{n}(U) \cap V \neq \emptyset, f$ is totally transitive if $f^{n}$ is transitive for any $n \geq 1, f$ is weakly mixing if $f \times f$ is transitive, and $f$ is mixing if for any nonempty open sets $U$ and $V$ there is an $N>0$ such that $f^{n}(U) \cap V \neq \emptyset$ for all $n \geq N$. Observe that $f$ is transitive, provided that $N(U, V)$ is infinite for any two nonempty open sets $U, V$, and mixing when $N(U, V)$ is cofinite for any two nonempty open sets $U, V$. If $\omega(f, x)=X$ then we say that $x$ is a transitive point.

A dynamical system $(X, f)$ has dense small periodic sets if for every nonempty open set $U \subset X$ there is a nonempty closed subset $A \subset U$ and $n>0$ such that $f^{n}(A)=A$. The later property was introduced by Huang and Ye [20] and it is closely related to the Smale's spectral decomposition for maps with shadowing (see [27], where it appears as property $(P))$. Note that when regularly recurrent points are dense then automatically $f$ has dense small periodic sets. When a system is minimal, then it has dense small periodic sets iff it has a dense set of regularly recurrent points iff it has a regularly recurrent point $[19,20]$. For more extensive comments on transitive systems with dense small periodic sets, the reader is referred to [22].

A point $x \in X$ in a dynamical system $(X, f)$ is sensitive if there is $\lambda>0$ such that for every nonempty open neighborhood $U$ of $x$ we have $\operatorname{diam}\left(f^{n}(U)\right)>\lambda$ for some $n>0$. A dynamical system $(X, f)$ is sensitive if there is $\lambda>0$ with the property that for every nonempty open set $U \subset X$, there is $n>0$ such that $\operatorname{diam}\left(f^{n}(U)\right)>\lambda$. In this case we say that $\lambda$ is a sensitivity constant for $f$, or that $f$ is $\lambda$-sensitive. Another extreme, completely opposite to sensitivity, is the notion of equicontinuity: $f$ is equicontinuous if for every $\lambda>0$, there is $\delta>0$ with the property that for every $a, b \in X$, condition $d(a, b)<\delta$ implies that $d\left(f^{n}(a), f^{n}(b)\right)<\lambda$ for every $n \in \mathbb{N}$.

The set $\left\{(x, y) \in X^{2}: \lim _{n \rightarrow \infty} d\left(f^{n}(x), f^{n}(y)\right)=0\right\}$ of all asymptotic pairs of $f$ is denoted as $A s y(f)$, and the set $\left\{(x, y) \in X^{2}: \liminf _{n \rightarrow \infty} d\left(f^{n}(x), f^{n}(y)\right)=0\right\}$ of all proximal pairs of $f$ is denoted as $\operatorname{Prox}(f)$. Note that if $(x, y) \in \operatorname{Prox}(f)$ then $\left\{n \in \mathbb{N}: d\left(f^{n}(x), f^{n}(y)\right)<\varepsilon\right\}$ is thick for every $\varepsilon>0$. Consequently, $\operatorname{Prox}\left(f^{k}\right)=$ $\operatorname{Prox}(f)$ for every $k \in \mathbb{N}$. Also $\operatorname{Asy}\left(f^{k}\right)=\operatorname{Asy}(f)$. A pair $(x, y)$ is distal if it is not proximal. A dynamical system $(X, f)$ is distal if $\operatorname{Prox}(f)$ reduces to the diagonal of $X^{2}$, and semi-distal if $R(f \times f) \cap[\operatorname{Prox}(f) \backslash A s y(f)]=\emptyset$. For more information about asymptoticity, proximality and distality, the reader is referred to the books $[2,4]$.

Let $(X, f)$ be a dynamical system. Fix any $\varepsilon>0$ and $\delta>0$. A sequence $\left\{x_{n}\right\}_{n=0}^{\infty} \subset$ $X$ is a $\delta$-pseudo orbit if $d\left(f\left(x_{n}\right), x_{n+1}\right)<\delta$ for $n=0,1,2, \ldots$; a point $z \in X$ is $\varepsilon$-tracing a pseudo-orbit $\left\{x_{n}\right\}_{n=0}^{\infty}$ when $d\left(f^{n}(z), x_{n}\right)<\varepsilon$ for all $n=0,1, \ldots$ We say that $\phi:(0, \infty) \rightarrow(0, \infty)$ is a tracing function for $f$ if:

(i) $\phi(\varepsilon)<\varepsilon$ for every $\varepsilon \in(0, \infty)$, and

(ii) every $\phi(\varepsilon)$-pseudo orbit of $f$ is $\varepsilon$-traced by some point in $X$. 
A map $f$ has the shadowing property (or pseudo-orbit tracing property) if there exists a tracing function for $f$. In other words, $f$ has shadowing property if for any $\varepsilon>0$ there is $\delta>0$ such that every $\delta$-pseudo orbit is $\varepsilon$-traced by a point in $X$. Note that if $\phi:(0, \infty) \rightarrow(0, \infty)$ is a tracing function for $f$, then any $\psi:(0, \infty) \rightarrow(0, \infty)$ with $\psi \leq \phi$ is also a tracing function for $f$.

A set $D \subset X$ is $f$-invariant (or simply invariant) if $f(D) \subset D$. A nonempty invariant set $D$ is minimal, if $\overline{O(f, x)}=D$ for every $x \in D$. It was first proved by Birkhoff (c.f. p. 93 of [13]) that a point $x$ is minimal if and only if $\overline{O(f, x)}$ is a minimal set.

Let $(X, f)$ and $(Y, g)$ be dynamical systems. If there is a continuous surjection $\pi: X \rightarrow Y$ with $\pi \circ f=g \circ \pi$ then we say that $\pi$ is a factor map, the system $(Y, g)$ is a factor of $(X, f)$ and $(X, f)$ is an extension of $(Y, g)$. If $\pi:(X, f) \rightarrow(Y, g)$ is a factor map and if $Z:=\left\{x \in X: \# \pi^{-1}(\pi(x))=1\right\}$ is residual in $X$, then $(X, f)$ is called an almost $1-1$ extension of $(Y, g)$, and $\pi^{-1}(y)$ is called a singleton fibre for any $y \in Y$ with $\# \pi^{-1}(y)=1$. If we also have $Z=X$, i.e., if $\pi$ is also a homeomorphism, then we say that $\pi$ is a conjugacy and the dynamical systems $(X, f)$, $(Y, g)$ are conjugate. Conjugate dynamical systems can be considered the same from the dynamical point of view.

\subsection{Expansive systems}

The classical definition of expansivity is given for invertible systems. It can be stated as follows. A homeomorphism $f: X \rightarrow X$ is expansive if there a constant $b>0$ (called an expansive constant) such that for any distinct $x, y \in X$ there is $n \in \mathbb{Z}$ such that $d\left(f^{n}(x), f^{n}(y)\right) \geq b$. In the case of non-invertible dynamical systems, there are a few possibilities to extend this definition. The classical approach is to define expansivity in terms of the acting (semi)-group. This way we obtain the definition applicable to all actions of topological groups, which in case of continuous surjections takes the following form. A dynamical system $(X, f)$ is positively expansive if there exists $b>0$ such that for any $x, y \in X, x \neq y$ there is $n \geq 0$ such that $d\left(f^{n}(x), f^{n}(y)\right) \geq$ $b$.

But in the case of continuous surjections there is another possibility. In place of expansivity of the action of $f$ we may check expansivity of the natural shift homeomorphism on the inverse limit of $f$. We present this definition after [6]. A continuous surjection $f: X \rightarrow X$ is $c$-expansive if there is a constant $b>0$ such that if for any $x, y \in X$ and any two full orbits $\left\{x_{n}\right\}_{n \in \mathbb{Z}},\left\{y_{n}\right\}_{n \in \mathbb{Z}}$ through $x$ and $y$ respectively, the condition

$$
d\left(x_{n}, y_{n}\right)<b \quad \text { for all } n \in \mathbb{Z}
$$

implies that $x=y$, where by a full orbit thorough $x$ (resp. $y$ ) we mean any sequence $\left\{z_{n}\right\}_{n \in \mathbb{Z}}$ such that $f\left(z_{n}\right)=z_{n+1}$ for every $n \in \mathbb{Z}$ and $z_{0}=x$ (resp. $z_{0}=y$ ).

Clearly, in the case of homeomorphisms expansivity and c-expansivity are the same property. A positively expansive map is always c-expansive but not vice-versa. 


\subsection{Odometers}

We briefly present the definition of an odometer (also called an adding machine) which is an important example of minimal equicontinuous system. Let $s=\left(s_{j}\right)_{j=1}^{\infty}$ be a strictly increasing sequence of positive integers such that $s_{1} \geq 2$ and $s_{j}$ divides $s_{j+1}$. Let $X(j)=\left\{0,1, \ldots, s_{j}-1\right\}$ and $X_{s}=\left\{x \in \prod_{j=1}^{\infty} X(j)\right.$ : $\left.x_{j+1} \equiv x_{j}\left(\bmod s_{j}\right)\right\}$. Then $X_{s}$ is a Cantor space with respect to the subspace topology (where $\prod_{j=1}^{\infty} X(j)$ has the product topology). Let $f: X_{s} \ni \quad x \mapsto$ $y \in X_{s}$, where $y_{j}=x_{j}+1\left(\bmod s_{j}\right)$ for each $j=1,2, \ldots$ The dynamical system $\left(X_{s}, f\right)$ is called an odometer defined by the sequence $s=\left\{s_{j}\right\}_{j=1}^{\infty}$. Note that $f$ is an invertible isometry with respect to a suitable metric on $X_{s}$, and in particular $f$ is equicontinuous. It may also be seen that $\left(X_{s}, f\right)$ is minimal but not totally transitive. Minimal equicontinuous maps of Cantor spaces are conjugate to odometers [1]; see the survey [16] for more details about odometers.

It can be proved (e.g. see [16, Theorem 5.1]) that a minimal system $D$ obtained as the closure $D=\overline{O(f, x)}$ of the orbit of a regularly recurrent point $x \in R R(f)$ is an almost 1-1 extension of an odometer. Furthermore, singleton fibers coincide with the set $R R(f) \cap D$, so $\left(D,\left.f\right|_{D}\right)$ is conjugate to an odometer exactly when $D=D \cap R R(f)$.

\subsection{Entropy}

There are various (equivalent) definitions of topological entropy for dynamical systems on compact metric spaces. Here we will follow the approach introduced by R. Bowen. Let $K \subset X$ be a nonempty closed set. Given an integer $n>0$ and $\varepsilon>0$, we say that a set $A \subset K$ is $(K, n, \varepsilon)$-separated for $f$ if for each pair $x, y$ of distinct points in $A$, there is an integer $0 \leq k<n$ such that $d\left(f^{k}(x), f^{k}(y)\right)>\varepsilon$. Denote by $s(f, K, n, \varepsilon)$ the maximal possible cardinality of a $(K, n, \varepsilon)$-separated set for $f$ contained in $K$ (this number is always well defined by the compactness of $X$ ). Using the above quantity, we define the following limit

$$
h(f, K)=\lim _{\varepsilon \rightarrow 0}\left[\limsup _{n \rightarrow \infty} \frac{\log s(f, K, n, \varepsilon)}{n}\right]
$$

Note that $h(f, K)$ is always well defined, since for any two integers $n, m>0$ we have $s(f, K, n+m, \varepsilon) \leq s(f, K, n, \varepsilon) s(f, K, m, \varepsilon)$ and $s(f, K, n, \varepsilon)$ is a non-increasing function of $\varepsilon>0$. The topological entropy of $f$ is defined as $h(f)=h(f, X)$. We say that $x \in X$ is an entropy point if $h(f, \bar{U})>0$ for every open neighborhood $U$ of $x$. Entropy points were introduced in [33] as natural elements of systems with positive topological entropy. We will denote by $E_{p}(f)$ the set of all entropy points of $f$. 


\subsection{Subshifts}

For any integer $m \geq 2$, the space $\{0,1, \ldots, m-1\}^{\mathbb{N}}$ is a Cantor space with respect to the product topology. A metric inducing the product topology on this space is

$$
d\left(\left\{x_{n}\right\}_{n=1}^{\infty},\left\{y_{n}\right\}_{n=1}^{\infty}\right)=\sum_{n=1}^{\infty} 2^{-n} \min \left\{1,\left|x_{n}-y_{n}\right|\right\}
$$

The shift map $\sigma:\{0,1, \ldots, m-1\}^{\mathbb{N}} \rightarrow\{0,1, \ldots, m-1\}^{\mathbb{N}}$ is defined by the condition that $(\sigma(x))_{n}=x_{n+1}$ for $n \in \mathbb{N}$. It is a continuous surjection and $\left(\{0,1, \ldots, m-1\}^{\mathbb{N}}, \sigma\right)$ is called the shift dynamical system. Clearly one may use any finite set with at least two members in the place of $\{0,1, \ldots, m-1\}$. If $X \subset\{0,1, \ldots, m-1\}^{\mathbb{N}}$ is a $\sigma$-invariant nonempty closed subset, then the subsystem $(X, \sigma)$ is called a subshift. A member $w$ of $\{0,1, \ldots, m-1\}^{n}$ is said to be a word of length $n$ over the alphabet $\{0,1, \ldots, m-1\}$ and we write $|w|=n$. If $(X, \sigma)$ is a subshift, then its language $L(X)$ is the collection of all words $w$ (including the empty word) appearing in some $x \in X$.

The above objects are defined analogously, when we consider two-sided full shift $\{0,1, \ldots, m-1\}^{\mathbb{Z}}$. The only difference now is that

$$
d\left(\left\{x_{n}\right\}_{n \in \mathbb{Z}},\left\{y_{n}\right\}_{n \in \mathbb{Z}}\right)=\sum_{n \in \mathbb{Z}} 2^{-|n|} \min \left\{1,\left|x_{n}-y_{n}\right|\right\} .
$$

\section{Small minimal sets and density of $R R(f)$}

As the first result, we show that shadowing implies the existence of small minimal sets for a sequence of powers of $f$, and moreover these minimal sets can be chosen to form certain periodic cycles modulo some prescribed error.

Lemma 3.1 Let $(X, f)$ be a non-wandering dynamical system having shadowing. Let $u \in X, \lambda>0$, and let $\left\{\lambda_{k}\right\}_{k=1}^{\infty}$ be a sequence of positive reals with $\sum_{k=1}^{\infty} \lambda_{k}<\lambda$. Then there exist an increasing sequence $\left\{m_{k}\right\}_{k=1}^{\infty}$ of natural numbers and a sequence $\left\{A_{k}\right\}_{k=1}^{\infty}$ of subsets of $X$ such that for every $k \in \mathbb{N}$ we have:

(1) $m_{k}$ divides $m_{k+1}$.

(2) $A_{k}$ is a minimal set for $f^{m_{k}}$ and $\operatorname{diam}\left(A_{k}\right) \leq \lambda_{k}$.

(3) $A_{k} \subset B\left(u, \sum_{j=1}^{k} \lambda_{j}\right) \subset B(u, \lambda)$.

(4) $f^{m_{j} n}\left(A_{k}\right) \subset B\left(A_{j}, \sum_{i=j}^{k} \lambda_{i}\right)$ for $1 \leq j \leq k$ and every $n \geq 0$.

Proof Let $\phi:(0, \infty) \rightarrow(0, \infty)$ be a tracing function for $f$ given by the shadowing property, and let $\varepsilon_{k}=4^{-1} \lambda_{k}$ for $k \in \mathbb{N}$. We use induction on $k$ to prove the lemma. Even though the technical details are a little complicated, the basic idea on which the proof is built is relatively simple: start with a minimal point, use it to form a periodic pseudo orbit, then choose a minimal point from the $\omega$-limit set of a point tracing this pseudo orbit, and repeat the process. 
The initial step of induction is as follows. By shadowing, minimal points are dense in $X$ by [25, Corollary 1]. Let $z_{0} \in M(f) \cap B\left(u, \varepsilon_{1}\right)$ and choose $m_{1} \in \mathbb{N}$ such that $d\left(z_{0}, f^{m_{1}}\left(z_{0}\right)\right)<\phi\left(\varepsilon_{1}\right)$. Let $y_{1} \in X$ be a point $\varepsilon_{1}$-tracing the periodic $\phi\left(\varepsilon_{1}\right)$-pseudo orbit

$$
\xi_{0}=\left(z_{0}, f\left(z_{0}\right), \ldots, f^{m_{1}-1}\left(z_{0}\right), z_{0}, f\left(z_{0}\right), \ldots, f^{m_{1}-1}\left(z_{0}\right), z_{0}, \ldots\right) .
$$

Then for every $n \geq 0$ we have $f^{m_{1} n}\left(y_{1}\right) \in \bar{B}\left(z_{0}, \varepsilon_{1}\right)$. Let $A_{1}$ be a minimal set of $f^{m_{1}}$ contained in $\omega\left(f^{\bar{m}_{1}}, y_{1}\right) \cap \bar{B}\left(z_{0}, \varepsilon_{1}\right) \subset B\left(u, \lambda_{1}\right)$. Since $\operatorname{diam}\left(A_{1}\right) \leq \operatorname{diam} \bar{B}\left(z_{0}, \varepsilon_{1}\right) \leq$ $2 \varepsilon_{1}<\lambda_{1}$, the first step of induction is completed.

Now fix $k \in \mathbb{N}$ and assume that $m_{j}, A_{j}$ have been chosen as required for $1 \leq j \leq k$. We describe below how to choose $m_{k+1}$ and $A_{k+1}$. Consider $z_{k} \in A_{k}$. Since $z_{k}$ is also a minimal point for $f^{m_{k}}$ (e.g. see [18, Theorem 9.11(iv)]), we can find a multiple $m_{k+1}$ of $m_{k}$ such that $d\left(z_{k}, f^{m_{k+1}}\left(z_{k}\right)\right)<\phi\left(\varepsilon_{k+1}\right)$. Let $y_{k+1} \in X$ be a point $\varepsilon_{k+1}$-tracing the periodic $\phi\left(\varepsilon_{k+1}\right)$-pseudo orbit

$$
\xi_{k}=\left(z_{k}, f\left(z_{k}\right), \ldots, f^{m_{k+1}-1}\left(z_{k}\right), z_{k}, f\left(z_{k}\right), \ldots, f^{m_{k+1}-1}\left(z_{k}\right), z_{k}, \ldots\right) .
$$

Then for every $n \geq 0$ we have $f^{m_{k+1} n}\left(y_{k+1}\right) \in \bar{B}\left(z_{k}, \varepsilon_{k+1}\right)$. Let $A_{k+1}$ be a minimal set of $f^{m_{k+1}}$ contained in $\omega\left(f^{m_{k+1}}, y_{k+1}\right) \cap \bar{B}\left(z_{k}, \varepsilon_{k+1}\right)$. Since $z_{k} \in A_{k} \subset B\left(u, \sum_{j=1}^{k} \lambda_{j}\right)$ by induction assumption together with inequality $\varepsilon_{k+1}<\lambda_{k+1}$, we have $A_{k+1} \subset$ $B\left(u, \sum_{j=1}^{k+1} \lambda_{j}\right) \subset B(u, \lambda)$. Also diam $\left(A_{k+1}\right) \leq \operatorname{diam} \bar{B}\left(z_{k}, \varepsilon_{k+1}\right) \leq 2 \varepsilon_{k+1}<\lambda_{k+1}$. Now it remains to prove assertion (4).

Fix any $x \in A_{k+1}, n \geq 0$ and $1 \leq j \leq k+1$. We need to show that $f^{m_{j} n}(x) \in B\left(A_{j}, \sum_{i=j}^{k+1} \lambda_{i}\right)$. Since $x \in A_{k+1} \subset \omega\left(f^{m_{k+1}}, y_{k+1}\right)$ and since $m_{j}$ divides $m_{k+1}$, there is an increasing sequence $\left\{q_{l}\right\}_{l=1}^{\infty}$ of positive integers such that $x=\lim _{l \rightarrow \infty} f^{m_{j} q_{l}}\left(y_{k+1}\right)$. Hence $f^{m_{j} n}(x)=\lim _{l \rightarrow \infty} f^{m_{j}\left(n+q_{l}\right)}\left(y_{k+1}\right)$. Fix $l \in \mathbb{N}$ and let $q=n+q_{l}$. There is $0 \leq p \leq m_{k+1} / m_{j}$ such that $m_{j} p \equiv m_{j} q\left(\bmod m_{k+1}\right)$.

Then by the choice of $y_{k+1}$, we get that $f^{m_{j} q}\left(y_{k+1}\right) \in \bar{B}\left(f^{m_{j} p}\left(z_{k}\right), \varepsilon_{k+1}\right)$. But $f^{m_{j} p}\left(z_{k}\right) \in f^{m_{j} p}\left(A_{k}\right) \subset B\left(A_{j}, \sum_{i=j}^{k} \lambda_{i}\right)$ by induction assumption, and therefore

$$
\begin{aligned}
& f^{m_{j}\left(n+q_{l}\right)}\left(y_{k+1}\right) \\
& \quad=f^{m_{j} q}\left(y_{k+1}\right) \in \bar{B}\left(B\left(A_{j}, \sum_{i=j}^{k} \lambda_{i}\right), \varepsilon_{k+1}\right) \subset \bar{B}\left(A_{j}, \sum_{i=j}^{k} \lambda_{i}+\varepsilon_{k+1}\right) .
\end{aligned}
$$

It follows that $f^{m_{j} n}(x) \in \bar{B}\left(A_{j}, \sum_{i=j}^{k} \lambda_{i}+\varepsilon_{k+1}\right) \subset B\left(A_{j}, \sum_{i=j}^{k+1} \lambda_{i}\right)$, completing the proof.

In [25] the author asked the following question:

- When can we say that $R R(f)$ is dense for a dynamical system having shadowing?

We answer it below by deducing density of the set of regularly recurrent points from the existence of small minimal sets in Lemma 3.1.

Theorem 3.2 Let $(X, f)$ be a non-wandering dynamical system having shadowing. Then the set $R R(f)$ is dense in $X$. 
Proof Let $U \subset X$ be nonempty and open. We need to find a point $z \in U \cap R R(f)$. Choose $u \in U$ and $\lambda>0$ such that $\bar{B}(u, \lambda) \subset U$. If we put $\lambda_{k}=4^{-k} \lambda$, then $\sum_{k=1}^{\infty} \lambda_{k}<\lambda$, and therefore there exist positive integers $m_{k}$ and $f^{m_{k}}$-minimal sets $A_{k}$ for every $k \in \mathbb{N}$ as specified by Lemma 3.1. Pick a point $z_{k} \in A_{k}$ for each $k \in \mathbb{N}$. If $k_{1}, k_{2} \geq j$, then both $z_{k_{1}}$ and $z_{k_{2}}$ belong to $B\left(A_{j}, \sum_{i=j}^{\infty} \lambda_{i}\right)$ by assertion (4) of Lemma 3.1. Since $\operatorname{diam}\left(A_{j}\right) \leq \lambda_{j}$, we have

$$
d\left(z_{k_{1}}, z_{k_{2}}\right) \leq \lambda_{j}+2 \sum_{i=j}^{\infty} \lambda_{i} \leq 3 \sum_{i=j}^{\infty} \lambda_{i}
$$

But $\lim _{j \rightarrow \infty} \sum_{i=j}^{\infty} \lambda_{i}=0$, which shows that $\left\{z_{k}\right\}_{k=1}^{\infty}$ is a Cauchy sequence, and hence we can define $z=\lim _{k \rightarrow \infty} z_{k}$. Since $z_{k} \in A_{k} \subset B(u, \lambda)$ for every $k \in \mathbb{N}$, we have $z \in \bar{B}(u, \lambda) \subset U$. We now claim that $z \in R R(f)$.

For any $j \in \mathbb{N}$ and $n \geq 0$, by Lemma 3.1(4) we have

$$
f^{m_{j} n}(z)=\lim _{k \rightarrow \infty} f^{m_{j} n}\left(z_{k}\right) \in \bar{B}\left(A_{j}, \sum_{i=j}^{\infty} \lambda_{i}\right)
$$

and therefore

$$
d\left(z, f^{m_{j} n}(z)\right) \leq \operatorname{diam}\left(A_{j}\right)+2 \sum_{i=j}^{\infty} \lambda_{i} \leq 3 \sum_{i=j}^{\infty} \lambda_{i}
$$

It follows that for any given $\beta>0$, there is $j \in \mathbb{N}$ such that $d\left(z, f^{m_{j} n}(z)\right)<\beta$ for every $n \in \mathbb{N}$. This proves our claim that $z \in R R(f)$, and completes the proof.

Corollary 3.3 Let $(X, f)$ be a dynamical system having shadowing. Then $R R(f)$ is dense in the non-wandering set $\Omega(f)$.

Proof By Lemma 1 and Corollary 1 of [25] (see also [6, Theorem 3.4.2]), $\left.f\right|_{\Omega}(f)$ is a non-wandering map having shadowing. Hence Theorem 3.2 applies.

\section{Density of $M(f) \backslash R R(f)$ and $R R(f) \backslash P(f)$}

We know by [25] that minimal points are dense in non-wandering dynamical systems having shadowing. If the system $(X, f)$ is also sensitive, we can improve the conclusion and show that both $M(f) \backslash R R(f)$ and $R R(f) \backslash P(f)$ are dense in $X$. For this purpose we need Lemma 4.1.

Lemma 4.1 The following assertions hold:

(i) There are pairwise disjoint syndetic sets $S_{1}, S_{2}, \ldots \subset \mathbb{N}$ with the property that $S_{k} \subset k \mathbb{N}$ for every integer $k \in \mathbb{N}$.

(ii) There is $x \in\{0,1\}^{\mathbb{N}}$ with the following properties: 
(a) for every $k, q \in \mathbb{N}$ and every thick set $T \subset \mathbb{N}$, there is an integer s such that $[s, s+q] \cap \mathbb{N} \subset T$ and $x_{k s}=0, x_{k(s+1)}=1$.

(b) for every $k, m, q \in \mathbb{N}$ and every thick set $T \subset \mathbb{N}$, there is an integer s such that $[k m s, k m(s+q)] \cap \mathbb{N} \subset T$ and $x_{k s}=0, x_{k(s+1)}=1$.

Proof (i) : It is enough to put $S_{k}=$ all odd multiples of $\left.k ! 2^{k}\right\}$ for $k=1,2, \ldots$ Simply, if we take any pair of integers $0<l<k$ and any $j \in S_{k}$ then there is an odd integer $n$ such that

$$
j=n(k !) 2^{k}=(l !) 2^{l}\left(n \frac{k !}{l !} 2^{k-l}\right) \notin S_{l} .
$$

(ii) : Let $S_{1}, S_{2}, \ldots$ be as above. Consider $n \in \mathbb{N}$. If $n$ does not belong to any $S_{k}$, we put $x_{n}=1$. Otherwise, there is a unique $k \in \mathbb{N}$ such that $n \in S_{k}$. Define $x_{n}=0$ or $x_{n}=1$, respectively, depending on whether $n$ occupies an odd or even position in $S_{k}$ when the members of $S_{k}$ are arranged as an increasing sequence.

Now consider a thick set $T \subset \mathbb{N}$ and $k, q \in \mathbb{N}$. Let $\widetilde{S_{k}}=S_{k} / k=\left\{n / k: n \in S_{k}\right\}$. Since $\widetilde{S_{k}}$ is syndetic and $T$ is thick, we can find integers $s_{1}<\cdots<s_{q+1}$ in $\widetilde{S_{k}} \cap T$ with the following properties: $\left[s_{1}, s_{q+1}\right] \cap \mathbb{N} \subset T$, the integer $k s_{1}$ occupies an odd position and $k s_{2}$ occupies an even position in $S_{k}$ when the members of $S_{k}$ are arranged in increasing order. Then $x_{k s_{1}}=0$ and $x_{k s_{2}}=1$ by our definition of $x$. Let $s \in\left[s_{1}, s_{2}\right) \cap \mathbb{N}$ be the largest integer with $x_{k s}=0$. Then $x_{k(s+1)}=1$. Moreover, $[s, s+q] \cap \mathbb{N} \subset\left[s_{1}, s_{q+1}\right] \cap \mathbb{N} \subset T$. This proves part (a). For part (b), apply part (a) to the thick set $T^{\prime}:=\{s \in \mathbb{N}:[k m s, k m(s+q)] \cap \mathbb{N} \subset T\}$.

Now we are ready to prove the promised result. We will also establish the existence of minimal sensitive subsystems, and this will be crucial for obtaining the full shift as a factor for some power of $f$ in the next section.

Theorem 4.2 Let $(X, f)$ be a non-wandering dynamical system having shadowing. If $f$ is sensitive, then for any nonempty open set $U \subset X$, there is a regularly recurrent point $z \in U$ such that the subsystem $(\overline{O(f, z)}, f)$ is sensitive. In particular $z$ is not a periodic point.

Proof The proof will extend techniques introduced in Theorem 3.2. The long proof is divided into four steps for the convenience of the reader. In the first step we state a claim, and the second step proves the theorem assuming the claim. Then we prove the claim using induction in steps three and four.

Step-1: Let $\phi:(0, \infty) \rightarrow(0, \infty)$ be a tracing function for $f$. Choose $\lambda>0$ and $u \in U$ such that $f$ is $2 \lambda$-sensitive and $\bar{B}(u, \lambda) \subset U$. Let $\lambda_{k}=4^{-k} \lambda$ for $k \in \mathbb{N}$. We claim that there exist a sequence $\left\{A_{k}\right\}_{k=1}^{\infty}$ of subsets of $X$, a sequence $\left\{z_{k}\right\}_{k=1}^{\infty}$ of points in $X$, and three increasing sequences $\left\{m_{k}\right\}_{k=1}^{\infty},\left\{m_{k}^{\prime}\right\}_{k=1}^{\infty},\left\{m_{k}^{\prime \prime}\right\}_{k=1}^{\infty}$ of positive integers such that for every $k \in \mathbb{N}$ we have:

(1) $k$ divides $m_{k}$, and $m_{k}$ divides $m_{k+1}$.

(2) $A_{k}$ is a minimal set for $f^{m_{k}}$, and $\operatorname{diam}\left(A_{k}\right) \leq \lambda_{k}$.

(3) $z_{k} \in A_{k} \subset B\left(u, \sum_{j=1}^{k} \lambda_{j}\right) \subset B(u, \lambda)$. 
(4) $f^{m_{j} n}\left(A_{k}\right) \subset B\left(A_{j}, \sum_{i=j}^{k} \lambda_{i}\right)$ for $1 \leq j \leq k$ and every $n \geq 0$.

(5) $m_{k}^{\prime}<m_{k}^{\prime \prime}$, and $m_{k}$ divides $m_{k}^{\prime \prime}-m_{k}^{\prime}$.

(6) $d\left(f^{m_{j}^{\prime}}\left(z_{k}\right), f^{m_{j}^{\prime \prime}}\left(z_{k}\right)\right)>2^{-1} \lambda$ for $1 \leq j \leq k$.

(7) For every $\gamma>0$, there are positive integer $l, r^{\prime}$ (depending on $\gamma$ ) such that $d\left(z_{k}, f^{l m_{k}}\left(z_{k}\right)\right)<\gamma$ and $d\left(f^{r^{\prime}}\left(z_{k}\right), f^{l m_{k}+r^{\prime}}\left(z_{k}\right)\right)>\lambda+\lambda_{k}$.

Step-2: Suppose first that the claim holds. As in the proof of Theorem 3.2, we may check that $\left\{z_{k}\right\}_{k=1}^{\infty}$ is a Cauchy sequence in $X$ and $z:=\lim _{k \rightarrow \infty} z_{k} \in \bar{B}(u, \lambda) \cap$ $R R(f) \subset U \cap R R(f)$. Let $K=\overline{O(f, z)}$. We need to show that the subsystem $(K, f)$ is sensitive. If $(K, f)$ is not sensitive, then it must be equicontinuous by minimality [3]. Choose $\beta>0$ so that $d\left(z^{\prime}, z^{\prime \prime}\right)<\beta$ implies $d\left(f^{n}\left(z^{\prime}\right), f^{n}\left(z^{\prime \prime}\right)\right)<2^{-1} \lambda$ for every $z^{\prime}, z^{\prime \prime} \in K$ and every $n \in \mathbb{N}$. Since $z$ is regularly recurrent, there is $p \in \mathbb{N}$ such that $d\left(z, f^{n p}(z)\right)<\beta$ for every $n \in \mathbb{N}$. Hence by the choice of $\beta$, we have $d\left(f^{m_{p}^{\prime}}(z), f^{m_{p}^{\prime \prime}}(z)\right)<2^{-1} \lambda$ since $p$ divides $m_{p}^{\prime \prime}-m_{p}^{\prime}$ by assertion (5) of our claim. On the other hand, by (6) we have $d\left(f^{m_{p}^{\prime}}(z), f^{m_{p}^{\prime \prime}}(z)\right)=$ $\lim _{k \rightarrow \infty} d\left(f^{m_{p}^{\prime}}\left(z_{k}\right), f^{m_{p}^{\prime \prime}}\left(z_{k}\right)\right) \geq 2^{-1} \lambda$, a contradiction. Hence $(K, f)$ must be sensitive.

It remains to prove the claim. We will prove it by induction on $k$. Let $\varepsilon=4^{-1} \lambda$ and $\varepsilon_{k}=4^{-1} \lambda_{k}$ for $k \in \mathbb{N}$. Note that $\varepsilon_{k}=4^{-k} \varepsilon$ for every $k \in \mathbb{N}$. Let $x \in\{0,1\}^{\mathbb{N}}$ be provided by Lemma 4.1(ii). We will make use of $x$ to construct pseudo orbits of special type at each step of the induction.

Step-3: The initial step of induction is done as follows. Let $z_{0}=u$. By the sensitivity of $f$ and density of $M(f \times f)$ in $X^{2}$, there are $a_{1}, b_{1} \in B\left(z_{0}, 4^{-1} \phi\left(\varepsilon_{1}\right)\right)$ and $r_{1} \in \mathbb{N}$ such that $\left(a_{1}, b_{1}\right) \in M(f \times f)$ and $d\left(f^{r_{1}}\left(a_{1}\right), f^{r_{1}}\left(b_{1}\right)\right)>2 \lambda=8 \varepsilon$. Let $m_{1}>r_{1}$ be an integer for which we have $d\left(a_{1}, f^{m_{1}}\left(a_{1}\right)\right)<4^{-1} \phi\left(\varepsilon_{1}\right)$ and $d\left(b_{1}, f^{m_{1}}\left(b_{1}\right)\right)<$ $4^{-1} \phi\left(\varepsilon_{1}\right)$. Define two finite sequences $\eta_{1}(0)$ and $\eta_{1}(1)$ in $X$ as follows:

$$
\begin{aligned}
& \eta_{1}(0)=\left(a_{1}, f\left(a_{1}\right), \ldots, f^{m_{1}-1}\left(a_{1}\right)\right), \\
& \eta_{1}(1)=\left(b_{1}, f\left(b_{1}\right), \ldots, f^{m_{1}-1}\left(b_{1}\right)\right) .
\end{aligned}
$$

Let $y_{1} \in X$ be a point $\varepsilon_{1}$-tracing the $\phi\left(\varepsilon_{1}\right)$-pseudo orbit

$$
\xi_{1}=\eta_{1}(0) \eta_{1}\left(x_{1}\right) \eta_{1}\left(x_{2}\right) \eta_{1}\left(x_{3}\right) \cdots
$$

Then for every $n \geq 0$ we have $f^{m_{1} n}\left(y_{1}\right) \in B\left(z_{0}, 4^{-1} \phi\left(\varepsilon_{1}\right)+\varepsilon_{1}\right) \subset B\left(z_{0}, 2 \varepsilon_{1}\right)$. Let $A_{1}$ be a minimal set of $f^{m_{1}}$ contained in $\omega\left(f^{m_{1}}, y_{1}\right) \cap \bar{B}\left(z_{0}, 2 \varepsilon_{1}\right) \subset B\left(u, \lambda_{1}\right) \subset$ $B(u, \lambda)$. We have $\operatorname{diam}\left(A_{1}\right) \leq \operatorname{diam} \bar{B}\left(z_{0}, 2 \varepsilon_{1}\right) \leq 4 \varepsilon_{1}=\lambda_{1}$. By Auslander-Ellis theorem (see [18, Proposition 8.6]) applied to $f^{m_{1}}$, we can find a point $z_{1} \in A_{1}$ such that $\left(y_{1}, z_{1}\right) \in \operatorname{Prox}\left(f^{m_{1}}\right)=\operatorname{Prox}(f)$. Since the set

$$
T_{1}:=\left\{n \in \mathbb{N}: d\left(f^{n}\left(y_{1}\right), f^{n}\left(z_{1}\right)\right)<\varepsilon_{1}\right\}
$$

is thick, we may find an integer $s_{1}>0$ such that $\left[m_{1} s_{1}, m_{1}\left(s_{1}+2\right)\right] \cap \mathbb{N} \subset T_{1}$ and $x_{s_{1}}=0$ and $x_{s_{1}+1}=1$, which is guaranteed by Lemma 4.1(ii) with $k=1$. Observe 
that by the definition of the $\phi\left(\varepsilon_{1}\right)$-pseudo orbit $\xi_{1}$, the $\left(m_{1} s_{1}+r_{1}\right)$ th term in $\xi_{1}$ is $f^{r_{1}}\left(a_{1}\right)$ and the $\left(m_{1}\left(s_{1}+1\right)+r_{1}\right)$ th term in $\xi_{1}$ is $f^{r_{1}}\left(b_{1}\right)$. Hence

$$
d\left(f^{r_{1}}\left(a_{1}\right), f^{m_{1} s_{1}+r_{1}}\left(y_{1}\right)\right) \leq \varepsilon_{1}, \quad \text { and } \quad d\left(f^{r_{1}}\left(b_{1}\right), f^{m_{1}\left(s_{1}+1\right)+r_{1}}\left(y_{1}\right)\right) \leq \varepsilon_{1} .
$$

Since $d\left(f^{r_{1}}\left(a_{1}\right), f^{r_{1}}\left(b_{1}\right)\right)>2 \lambda=8 \varepsilon$, we deduce that (recall that $\left.\varepsilon_{1}=4^{-1} \varepsilon\right)$ :

$$
d\left(f^{m_{1} s_{1}+r_{1}}\left(z_{1}\right), f^{m_{1}\left(s_{1}+1\right)+r_{1}}\left(z_{1}\right)\right)>8 \varepsilon-\left(\varepsilon_{1}+\varepsilon_{1}+\varepsilon_{1}+\varepsilon_{1}\right) \geq 7 \varepsilon>2^{-1} \lambda .
$$

Taking $m_{1}^{\prime}=m_{1} s_{1}+r_{1}$ and $m_{1}^{\prime \prime}=m_{1}\left(s_{1}+1\right)+r_{1}$, we see that (6) is satisfied.

To complete first step of induction, we need to show that (7) holds with $k=1$. Fix $\gamma>0$. Since $z_{1} \in M(f)=M\left(f^{m_{1}}\right)$, there is $l \in \mathbb{N}$ such that $d\left(z_{1}, f^{l m_{1}}\left(z_{1}\right)\right)<\gamma$. Since $T_{1}$ is thick, there is an integer $s$ such that $\left[\operatorname{lm}_{1} s, \operatorname{lm}_{1}(s+2)\right] \cap \mathbb{N} \subset T_{1}$, and $x_{l s}=0, x_{l(s+1)}=1$ by Lemma 4.1. Then for $r^{\prime}:=l m_{1} s+r_{1}$, we have that

$$
d\left(f^{r_{1}}\left(a_{1}\right), f^{r^{\prime}}\left(y_{1}\right)\right) \leq \varepsilon_{1}, \quad \text { and } \quad d\left(f^{r_{1}}\left(b_{1}\right), f^{l m_{1}+r^{\prime}}\left(y_{1}\right)\right) \leq \varepsilon_{1} .
$$

Since $r^{\prime}, \operatorname{lm}_{1}+r^{\prime} \in\left[\operatorname{lm}_{1} s, \operatorname{lm}_{1}(s+2)\right] \cap \mathbb{N} \subset T_{1}$, we also have

$$
d\left(f^{r^{\prime}}\left(y_{1}\right), f^{r^{\prime}}\left(z_{1}\right)\right)<\varepsilon_{1} \quad \text { and } \quad d\left(f^{l m_{1}+r^{\prime}}\left(y_{1}\right), f^{l m_{1}+r^{\prime}}\left(z_{1}\right)\right)<\varepsilon_{1} .
$$

Since $d\left(f^{r_{1}}\left(a_{1}\right), f^{r_{1}}\left(b_{1}\right)\right)>2 \lambda=8 \varepsilon$, we deduce as in the proof of assertion (6) that

$$
d\left(f^{r^{\prime}}\left(z_{1}\right), f^{l m_{1}+r^{\prime}}\left(z_{1}\right)\right)>8 \varepsilon-\left(\varepsilon_{1}+\varepsilon_{1}+\varepsilon_{1}+\varepsilon_{1}\right) \geq 7 \varepsilon>4 \varepsilon+\varepsilon=\lambda+\lambda_{1} .
$$

Indeed (7) holds, and so the first step of induction is completed.

Step-4: Now fix $k>1$, and assume that we have chosen $z_{j}, A_{j}, m_{j}, m_{j}^{\prime}, m_{j}^{\prime \prime}$ for $\overline{1 \leq j}<k$ as specified by conditions (1)-(7). We describe below how to choose $z_{k}, A_{k}, m_{k}, m_{k}^{\prime}$, and $m_{k}^{\prime \prime}$.

Let $\varepsilon_{k}^{\prime} \in\left(0, \varepsilon_{k}\right)$ be such that every point of $B\left(z_{k-1}, 4 \varepsilon_{k}^{\prime}\right)$ satisfies (6), that is if $v \in B\left(z_{k-1}, 4 \varepsilon_{k}^{\prime}\right)$ then $d\left(f^{m_{j}^{\prime}}(v), f^{m_{j}^{\prime \prime}}(v)\right)>2^{-1} \lambda$ for every $1 \leq j<k$.

By induction assumption, and to be specific, by assertion (7) of the claim there are $l^{\prime}, r_{k} \in \mathbb{N}$ such that the points $a_{k}:=z_{k-1}$ and $b_{k}:=f^{l^{\prime} m_{k-1}}\left(z_{k-1}\right)$ from $A_{k-1}$ satisfy

$$
d\left(a_{k}, b_{k}\right)<4^{-1} \phi\left(\varepsilon_{k}^{\prime}\right) \text { and } d\left(f^{r_{k}}\left(a_{k}\right), f^{r_{k}}\left(b_{k}\right)\right)>\lambda+\lambda_{k-1}>4 \varepsilon .
$$

Since $\left(a_{k}, b_{k}\right) \in M(f \times f)$, we can find an integer $m_{k}>r_{k}$ satisfying

$$
d\left(a_{k}, f^{m_{k}}\left(a_{k}\right)\right)<4^{-1} \phi\left(\varepsilon_{k}^{\prime}\right) \text { and } d\left(b_{k}, f^{m_{k}}\left(b_{k}\right)\right)<4^{-1} \phi\left(\varepsilon_{k}^{\prime}\right) .
$$

Since $M(f \times f)=M\left(f^{j} \times f^{j}\right)$ for every $j \in \mathbb{N}$, without loss of generality we may assume that both $k$ and $m_{k-1}$ divide $m_{k}$. Define two finite sequences $\eta_{k}(0)$ and $\eta_{k}(1)$ in $X$ as follows:

$$
\begin{aligned}
& \eta_{k}(0)=\left(a_{k}, f\left(a_{k}\right), \ldots, f^{m_{k}-1}\left(a_{k}\right)\right) \\
& \eta_{k}(1)=\left(b_{k}, f\left(b_{k}\right), \ldots, f^{m_{k}-1}\left(b_{k}\right)\right)
\end{aligned}
$$


Let $y_{k} \in X$ be a point $\varepsilon_{k}^{\prime}$-tracing the $\phi\left(\varepsilon_{k}^{\prime}\right)$-pseudo orbit

$$
\xi_{k}=\eta_{k}(0) \eta_{k}\left(x_{1}\right) \eta_{k}\left(x_{2}\right) \eta_{k}\left(x_{3}\right) \cdots
$$

Then for every $n \geq 0$ we have $f^{m_{k} n}\left(y_{k}\right) \in B\left(z_{k-1}, 4^{-1} \phi\left(\varepsilon_{k}^{\prime}\right)+\varepsilon_{k}^{\prime}\right) \subset B\left(z_{k-1}, 2 \varepsilon_{k}^{\prime}\right)$. Let $A_{k}$ be a minimal set of $f^{m_{k}}$ contained in $\omega\left(f^{m_{k}}, y_{k}\right) \subset \bar{B}\left(z_{k-1}, 2 \varepsilon_{k}^{\prime}\right)$. We have $\operatorname{diam}\left(A_{k}\right) \leq \operatorname{diam} \bar{B}\left(z_{k-1}, 2 \varepsilon_{k}^{\prime}\right) \leq 4 \varepsilon_{k}^{\prime}<4 \varepsilon_{k}=\lambda_{k}$. Note that $z_{k-1} \in A_{k-1} \subset$ $B\left(u, \sum_{j=1}^{k-1} \lambda_{j}\right)$ by induction assumption, and therefore $A_{k} \subset B\left(u, \sum_{j=1}^{k} \lambda_{j}\right) \subset$ $B(u, \lambda)$ also.

Observe that we have completed the proofs of assertions (1), (2), (3) in the $k$ th step of induction. The proof of (4) is as in the proof of Lemma 3.1. We present it for completeness.

Fix any $y \in A_{k}, n \geq 0$ and $1 \leq j \leq k$. We need to show $f^{m_{j} n}(y) \in$ $B\left(A_{j}, \sum_{i=j}^{k} \lambda_{i}\right)$. Since $y \in A_{k} \subset \omega\left(f^{m_{k}}, y_{k}\right)$ and since $m_{j}$ divides $m_{k}$, there is an increasing sequence $\left\{q_{l}\right\}_{l=1}^{\infty}$ of positive integers such that $y=\lim _{l \rightarrow \infty} f^{m_{j} q_{l}}\left(y_{k}\right)$. Hence $f^{m_{j} n}(y)=\lim _{l \rightarrow \infty} f^{m_{j}\left(n+q_{l}\right)}\left(y_{k}\right)$. Fix $l \in \mathbb{N}$ and let $q=n+q_{l}$. There is $0 \leq p \leq m_{k} / m_{j}$ such that $m_{j} p \equiv m_{j} q\left(\bmod m_{k}\right)$. Then by the choice of $y_{k}$, we get that either

$$
f^{m_{j} q}\left(y_{k}\right) \in \bar{B}\left(f^{m_{j} p}\left(a_{k}\right), \varepsilon_{k}\right), \quad \text { or } f^{m_{j} q}\left(y_{k}\right) \in \bar{B}\left(f^{m_{j} p}\left(b_{k}\right), \varepsilon_{k}\right) .
$$

But $a_{k}, b_{k} \in A_{k-1}$ by our choice, and $f^{m_{j} p}\left(A_{k-1}\right) \subset B\left(A_{j}, \sum_{i=j}^{k-1} \lambda_{i}\right)$ by induction assumption. Therefore

$$
\begin{aligned}
& f^{m_{j}\left(n+q_{l}\right)}\left(y_{k}\right) \\
& \quad=f^{m_{j} q}\left(y_{k}\right) \in \bar{B}\left(B\left(A_{j}, \sum_{i=j}^{k-1} \lambda_{i}\right), \varepsilon_{k}\right) \subset \bar{B}\left(A_{j}, \sum_{i=j}^{k-1} \lambda_{i}+\varepsilon_{k}\right) .
\end{aligned}
$$

It follows that $f^{m_{j} n}(y) \in \bar{B}\left(A_{j}, \sum_{i=j}^{k-1} \lambda_{i}+\varepsilon_{k}\right) \subset B\left(A_{j}, \sum_{i=j}^{k} \lambda_{i}\right)$, completing the proof of (4).

It remains to establish assertions (5)-(7). By Auslander-Ellis theorem (see [18, Proposition 8.6]) applied to $f^{m_{k}}$, we can find a point $z_{k} \in A_{k}$ such that $\left(y_{k}, z_{k}\right) \in$ $\operatorname{Prox}\left(f^{m_{k}}\right)=\operatorname{Prox}(f)$.

Since $z_{k} \in A_{k} \subset \bar{B}\left(z_{k-1}, 2 \varepsilon_{k}^{\prime}\right) \subset B\left(z_{k-1}, 4 \varepsilon_{k}^{\prime}\right)$, we obtain by the choice of $\varepsilon_{k}^{\prime}$ that

$$
d\left(f^{m_{j}^{\prime}}\left(z_{k}\right), f^{m_{j}^{\prime \prime}}\left(z_{k}\right)\right)>2^{-1} \lambda \quad \text { for every } 1 \leq j<k
$$

Since $T_{k}:=\left\{n \in \mathbb{N}: d\left(f^{n}\left(y_{k}\right), f^{n}\left(z_{k}\right)\right)<\varepsilon_{k}^{\prime}\right\}$ is thick, we may find by Lemma 4.1(ii) an integer $s_{k}>0$ such that $\left[s_{k} m_{k},\left(s_{k}+2\right) m_{k}\right] \subset T_{k}$ and additionally $x_{s_{k}}=0$ and $x_{s_{k}+1}=1$. Since $d\left(f^{r_{k}}\left(a_{k}\right), f^{r_{k}}\left(b_{k}\right)\right)>4 \varepsilon$ and $\varepsilon_{k}^{\prime}<\varepsilon_{k}<4^{-k} \varepsilon$, we may deduce the same way as in the initial step of induction that

$$
d\left(f^{m_{k} s_{k}+r_{k}}\left(z_{k}\right), f^{m_{k}\left(s_{k}+1\right)+r_{k}}\left(z_{k}\right)\right)>4 \varepsilon-\left(\varepsilon_{k}^{\prime}+\varepsilon_{k}^{\prime}+\varepsilon_{k}^{\prime}+\varepsilon_{k}^{\prime}\right)>3 \varepsilon>2^{-1} \lambda .
$$


Taking $m_{k}^{\prime}=m_{k} s_{k}+r_{k}$ and $m_{k}^{\prime \prime}=m_{k}\left(s_{k}+1\right)+r_{k}$, the proofs of (5) and (6) in the $k$ th step of induction are also completed.

As the last step, we need to prove (7). Fix any $\gamma>0$. Since $z_{k} \in M(f)=M\left(f^{m_{k}}\right)$, there is $l>0$ be such that $d\left(z_{k}, f^{l m_{k}}\left(z_{k}\right)\right)<\gamma$. Since $T_{k}$ is thick, there is an integer $s$ such that $\left[\operatorname{lm}_{k} s, \operatorname{lm}_{k}(s+2)\right] \cap \mathbb{N} \subset T_{k}$, and $x_{l s}=0, x_{l(s+1)}=1$ by Lemma 4.1. Then for $r^{\prime}:=l m_{k} s+r_{k}$, we have that

$$
d\left(f^{r_{k}}\left(a_{k}\right), f^{r^{\prime}}\left(y_{k}\right)\right) \leq \varepsilon_{k}^{\prime}, \quad \text { and } d\left(f^{r_{k}}\left(b_{k}\right), f^{l m_{k}+r^{\prime}}\left(y_{k}\right)\right) \leq \varepsilon_{k}^{\prime} \text {. }
$$

Since $r^{\prime}, \operatorname{lm}_{k}+r^{\prime} \in\left[\operatorname{lm}_{k} s, \operatorname{lm}_{k}(s+2)\right] \cap \mathbb{N} \subset T_{k}$, we also have

$$
d\left(f^{r^{\prime}}\left(y_{k}\right), f^{r^{\prime}}\left(z_{k}\right)\right)<\varepsilon_{k}^{\prime} \quad \text { and } d\left(f^{l m_{k}+r^{\prime}}\left(y_{k}\right), f^{l m_{k}+r^{\prime}}\left(z_{k}\right)\right)<\varepsilon_{k}^{\prime} .
$$

Since $4 \varepsilon_{k}^{\prime}<4 \varepsilon_{k}=\lambda_{k}=4^{-k} \lambda$ and since $d\left(f^{r_{k}}\left(a_{k}\right), f^{r_{k}}\left(b_{k}\right)\right)>\lambda+\lambda_{k-1}$, similarly as before we obtain that

$$
\begin{aligned}
d\left(f^{r^{\prime}}\left(z_{k}\right), f^{l m_{k}+r^{\prime}}\left(z_{k}\right)\right) & >\lambda+\lambda_{k-1}-4 \varepsilon_{k}^{\prime}>\left(1+4^{-(k-1)}-4^{-k}\right) \lambda \\
& >\left(1+4^{-k}\right) \lambda=\lambda+\lambda_{k} .
\end{aligned}
$$

Indeed (7) in the $k$-th step of induction holds. This completes the induction, proving the claim and ending the proof of theorem at the same time.

Corollary 4.3 Let $(X, f)$ be a non-wandering dynamical system having shadowing property. If $f$ is sensitive, then:

(1) $R R(f) \backslash P(f)$ is dense in $X$,

(2) $M(f) \backslash R R(f)$ is dense in $X$.

Proof Since a periodic orbit is an equicontinuous dynamical system, (1) is an immediate consequence of Theorem 4.2. For the proof of (2) fix a nonempty open set $U \subset X$ and use Theorem 4.2 to find $y \in R R(f) \cap U$ such that $D=\overline{O(f, y)}$ is sensitive. Then $D \backslash R R(f) \neq \varnothing$ as otherwise $\left(D,\left.f\right|_{D}\right)$ is conjugated to an odometer by [16, Theorem 5.1], and in particular is not sensitive. Fix $z \in D \backslash R R(f)$ and observe that there is $n \geq 0$ such that $z \in f^{-n}(U)$, since $D \subset \bigcup_{j=0}^{\infty} f^{-j}(U)$. Then $f^{n}(z) \in U$ which ends the proof since $f^{n}(z) \in M(f) \backslash R R(f)$.

Remark 4.4 Downarowicz and Ye [17] constructed a non-minimal dynamical system $(X, f)$ with property that every point is either transitive or periodic (and both types of points are present). Such a system cannot have shadowing by the above Corollary. This can also be deduced using Theorem 5.2 from the next section.

\section{Shift factor and minimal subsystems with positive entropy}

The presence of the shift dynamical system as a factor of some subsystem is an indication of high complexity for a dynamical system. A classical example of this type is Smale's horseshoe. In this section we will show that non-wandering, sensitive 
dynamical systems $(X, f)$ with shadowing have the property that the full shift is a factor for some subsystem of some power of $f$. In fact, we will show that such subsystems can be found everywhere in the phase space.

Theorem 5.1 Let $(X, f)$ be a dynamical system having shadowing, and $U \subset X$ be open. If there is a minimal sensitive subsystem $(M, f)$ with $M \cap U \neq \emptyset$, then there exist $m \in \mathbb{N}$, a subsystem $\left(Y, f^{m}\right)$ for $f^{m}$ with $Y \subset U$, and a factor map $\pi:\left(Y, f^{m}\right) \rightarrow\left(\{0,1\}^{\mathbb{N}}, \sigma\right)$.

Proof Let $u \in M \cap U$, and let $\lambda>0$ be a sensitivity constant for $(M, f)$ such that $\bar{B}(u, \lambda) \subset U$. Let $S:=N_{f}\left(u, B\left(u, 4^{-1} \lambda\right)\right)$. Write $S=\left\{0=s_{0}<s_{1}<s_{2}<\cdots\right\}$, and let $q \in \mathbb{N}$ be a bound for the gaps in the syndetic set $S$. Choose $\varepsilon>0$ with the property that $d(a, b) \leq 4 \varepsilon$ implies $d\left(f^{i}(a), f^{i}(b)\right)<2^{-1} \lambda$ for every $a, b \in X$ and $0 \leq i \leq q$. Keep in mind that $\varepsilon<8^{-1} \lambda$ by our choice.

Let $\phi:(0, \infty) \rightarrow(0, \infty)$ be a tracing function for $f$. By the minimality and sensitivity of $(M, f)$, there exist $r>q$ and $t \in \mathbb{N}$ such that $v:=f^{t}(u) \in B\left(u, 4^{-1} \phi(\varepsilon)\right)$ and $d\left(f^{r}(u), f^{r}(v)\right)>2^{-1} \lambda$. Then by the syndetic nature of $S$ and the choice of $\varepsilon$, there is at least one $s_{p} \in S$ such that $d\left(f^{s_{p}}(u), f^{s_{p}}(v)\right) \geq 4 \varepsilon$.

Now assume that $p \in \mathbb{N}$ is the smallest such that $s_{p} \in S$ satisfies $d\left(f^{s_{p}}(u)\right.$, $\left.f^{s_{p}}(v)\right) \geq 4 \varepsilon$. Then $d\left(f^{s_{p-1}}(u), f^{s_{p-1}}(v)\right)<4 \varepsilon$, and therefore $d\left(f^{s_{p}}(u), f^{s_{p}}(v)\right)<$ $2^{-1} \lambda$ by the choice of $\varepsilon$. We have $d\left(u, f^{s_{p}}(u)\right)<4^{-1} \lambda$ by the definition of $S$, and $d\left(u, f^{s_{p}}(v)\right) \leq d\left(u, f^{s_{p}}(u)\right)+d\left(f^{s_{p}}(u), f^{s_{p}}(v)\right)<4^{-1} \lambda+2^{-1} \lambda$. Therefore if we put $C(0)=\bar{B}\left(f^{s_{p}}(u), \varepsilon\right)$, and $C(1)=\bar{B}\left(f^{s_{p}}(v), \varepsilon\right)$, then $C(0) \cup C(1) \subset B(u, \lambda) \subset U$. Observe that $d\left(c_{0}, c_{1}\right) \geq 2 \varepsilon$ for every $\left(c_{0}, c_{1}\right) \in C(0) \times C(1)$, and in particular $C(0) \cap C(1)=\emptyset$. We will construct the required $Y$ as a subset of $C(0) \cup C(1)$.

Since $v=f^{t}(u)$, we have $(u, v) \in M(f \times f)$, and therefore there is an integer $m>s_{p}$ such that $d\left(u, f^{m}(u)\right)<4^{-1} \phi(\varepsilon)$ and $d\left(v, f^{m}(v)\right)<4^{-1} \phi(\varepsilon)$. Define two finite sequences

$$
\begin{aligned}
& \eta(0)=\left(u, f(u), \ldots, f^{m-1}(u)\right), \\
& \eta(1)=\left(v, f(v), \ldots, f^{m-1}(v)\right) .
\end{aligned}
$$

Fix a transitive point $x \in\{0,1\}^{\mathbb{N}}$ for the shift map $\sigma$, and let $y^{\prime} \in X$ be a point $\varepsilon$-tracing the $\phi(\varepsilon)$-pseudo orbit $\eta\left(x_{1}\right) \eta\left(x_{2}\right) \eta\left(x_{3}\right) \cdots$. If we put $y=f^{s_{p}}\left(y^{\prime}\right)$, then $f^{m n}(y) \in C\left(x_{n}\right)$ for every $n \geq 0$. Let $Y_{x}=\left\{f^{m n}(y): n=0,1,2, \ldots\right\}$ and $Y=$ $\overline{Y_{x}} \subset C(0) \cup C(1) \subset U$. Define $\pi: Y_{x} \rightarrow\{0,1\}^{\mathbb{N}}$ as $\pi\left(f^{m n}(y)\right)=\sigma^{n}(x)$ for $n \geq 0$. Note that $\pi\left(f^{m}(z)\right)=\sigma(\pi(z))$ for every $z \in Y_{x}$ by definition.

We claim that $\pi$ is uniformly continuous on $Y_{x}$. Given $k \in \mathbb{N}$, choose $\beta>0$ such that $d(a, b)<\beta$ implies $d\left(f^{i m}(a), f^{i m}(b)\right)<\varepsilon$ for every $a, b \in X$ and $0 \leq i \leq k$. Consider $z_{1}, z_{2} \in Y_{x}$ with $d\left(z_{1}, z_{2}\right)<\beta$. Let $n_{1}, n_{2} \geq 0$ be such that $z_{1}=f^{m n_{1}}(y)$ and $z_{2}=f^{m n_{2}}(y)$. We have $d\left(f^{m\left(n_{1}+i\right)}(y), f^{m\left(n_{2}+i\right)}(y)\right)<\varepsilon$ for $0 \leq i \leq k$ by the choice of $\beta$. Since $d\left(c_{0}, c_{1}\right) \geq 2 \varepsilon$ for any $\left(c_{0}, c_{1}\right) \in C(0) \times C(1)$, we conclude that both $f^{m\left(n_{1}+i\right)}(y)$ and $f^{m\left(n_{2}+i\right)}(y)$ are in $C\left(x_{n_{1}+i}\right)$ for $0 \leq i<k$. Consequently, $\pi\left(z_{1}\right)_{i}=\sigma^{n_{1}}(x)_{i}=\sigma^{n_{2}}(x)_{i}=\pi\left(z_{2}\right)_{i}$ for $1 \leq i \leq k$, and this establishes our claim. Therefore, $\pi$ extends to a continuous map $\pi: Y \rightarrow\{0,1\}^{\mathbb{N}}$ having the property that $\pi \circ f^{m}=\sigma \circ \pi$. Since the orbit of the transitive point $x$ is contained in the image of $\pi$, it follows that $\pi$ is surjective, and thus $\pi$ is a factor map. 
Theorem 5.2 Let $(X, f)$ be a non-wandering dynamical system having shadowing. Suppose $f$ is sensitive, and let $U \subset X$ be a nonempty open set. Then the following assertions hold.

(1) There exist $m \in \mathbb{N}$, a subsystem $\left(Y, f^{m}\right)$ for $f^{m}$ with $Y \subset U$, and a factor map $\pi:\left(Y, f^{m}\right) \rightarrow\left(\{0,1\}^{\mathbb{N}}, \sigma\right)$.

(2) There are $m \in \mathbb{N}$ and a minimal sensitive subsystem $\left(M, f^{m}\right)$ for $f^{m}$ such that $M \subset U, M \cap R R\left(f^{m}\right)=\emptyset$ and $h\left(\left.f^{m}\right|_{M}\right)>0$.

(3) There is a minimal sensitive subsystem $\left(M^{\prime}, f\right)$ for $f$ such that $M^{\prime} \cap U \neq \emptyset$, $M^{\prime} \cap R R(f)=\emptyset$ and $h\left(\left.f\right|_{M^{\prime}}\right)>0$.

Proof (1) : By Theorem 4.2, there is a minimal sensitive subsystem $(M, f)$ with $M \cap U \neq \emptyset$. Then by Theorem 5.1, there are $m \in \mathbb{N}$, a subsystem $\left(Y, f^{m}\right)$ for $f^{m}$ with $Y \subset U$, and a factor map $\pi:\left(Y, f^{m}\right) \rightarrow\left(\{0,1\}^{\mathbb{N}}, \sigma\right)$.

(2) : Let $m, Y, \pi$ be as above. Let $Q \subset\{0,1\}^{\mathbb{N}}$ be such that the subshift $(Q, \sigma)$ is minimal, has positive entropy and does not contain regularly recurrent points. For example we can start with an extension of Chacon subshift [12] which are formally subsystems of $\left(\{0, \ldots, k\}^{\mathbb{N}}, \sigma\right)$ for some $k>0$, hence can naturally viewed as a subsystem of $\left(\{0,1\}^{\mathbb{N}}, \sigma^{k}\right)$. But $M(\sigma) \cap R R(\sigma)=M\left(\sigma^{m}\right) \cap R R\left(\sigma^{m}\right)$, so it induces a minimal subshift $Q \subset\{0,1\}^{\mathbb{N}}$ with positive topological entropy.

Then $\pi^{-1}(Q)$ is an $f^{m}$-invariant closed set. Let $M \subset \pi^{-1}(Q) \subset Y \subset U$ be a minimal set for $f^{m}$. Then $\pi(M)=Q$ by the minimality of $(Q, \sigma)$, and therefore $h\left(\left.f^{m}\right|_{M}\right) \geq h\left(\left.\sigma\right|_{Q}\right)>0$. By the same argument $M \cap R R\left(f^{m}\right)=\emptyset$ (otherwise $Q$ would contain regularly recurrent points). It is also well-known that a minimal system with positive entropy must be sensitive (since equicontinuous systems have zero entropy).

(3) : Take $M^{\prime}=\bigcup_{j=0}^{m-1} f^{j}(M)$. Take any $y \in M^{\prime}$. Then $x \in M\left(f^{m}\right)=M(f)$ and

$$
\omega(f, x)=\bigcup_{j=0}^{m-1} \omega\left(f^{m}, f^{j}(x)\right)=\bigcup_{j=0}^{m-1} f^{j}\left(\omega\left(f^{m}, x\right)\right)=\bigcup_{j=0}^{m-1} f^{j}(M)=M^{\prime} .
$$

Indeed $M^{\prime}$ is a minimal set and

$$
h\left(\left.f\right|_{M^{\prime}}\right)=\frac{1}{m} h\left(\left.f^{m}\right|_{M^{\prime}}\right) \geq \frac{1}{m} h\left(\left.f^{m}\right|_{M}\right)>0 .
$$

Restrict $f$ to $M^{\prime}$. For any open set $U \subset M^{\prime}$ there is $s>0$ such that $M^{\prime}=$ $\bigcup_{j=0}^{s} f^{-j}(U)$, in particular there is $j \geq 0$ such that $f^{-j}(U) \cap M \neq \emptyset$. This immediately implies that $\left.f\right|_{M^{\prime}}$ is sensitive, since $M$ is sensitive. Finally, $M^{\prime} \cap$ $R R(f)=\emptyset$, as otherwise $M \cap R R(f) \neq \emptyset$ which is impossible.

Corollary 5.3 Let $(X, f)$ be a non-wandering dynamical system having shadowing. Suppose $f$ is sensitive, and let $U \subset X$ be a nonempty open set. Then there exist $\left(x_{i}, y_{i}\right) \in U \times U$ for $i=1,2,3$ such that

(1) $\left(x_{1}, y_{1}\right) \in \operatorname{Asy}(f)$ and $x_{1} \neq y_{1}$.

(2) $\left(x_{2}, y_{2}\right) \in \operatorname{Prox}(f) \backslash A \operatorname{ssy}(f)$. 
(3) $x_{3} \neq y_{3}$, both are distal points, and in particular $\left(x_{3}, y_{3}\right) \in X^{2} \backslash \operatorname{Prox}(f)$.

Proof By Theorem 5.2, there exist $m \in \mathbb{N}$ and a minimal sensitive subsystem $\left(M, f^{m}\right)$ for $f^{m}$ such that $M \subset U$ and $h\left(\left.f^{m}\right|_{M}\right)>0$. Since $h\left(\left.f^{m}\right|_{M}\right)>0$, we have that $\operatorname{Asy}\left(\left.f^{m}\right|_{M}\right)$ contains non-diagonal pairs by [11], and $\operatorname{Prox}\left(\left.f^{m}\right|_{M}\right) \backslash A s y\left(\left.f^{m}\right|_{M}\right) \neq \emptyset$ (in fact, uncountable) by [10]. But $A s y(f)=A s y\left(f^{m}\right)$, and $\operatorname{Prox}(f) \backslash A s y(f)=$ $\operatorname{Prox}\left(f^{m}\right) \backslash A s y\left(f^{m}\right)$. This proves (1) and (2).

By Theorem 4.3 there is a non-periodic, regularly recurrent (hence distal) point $x_{3} \in U$. The orbit of $x_{3}$ does not have any periodic or isolated points, and so there is another non-periodic, regularly recurrent point $y_{3} \in U \cap \overline{O\left(f, x_{3}\right)}$.

Note that by Theorem 3.2 the assertion (3) in Corollary 5.3 holds when $X$ has no isolated points, irrespective of whether $f$ is sensitive or not.

Dynamical system $(X, f)$ with surjective $f$ which is c-expansive and has shadowing property is usually called topologically hyperbolic, since it mimics many properties of hyperbolic systems in smooth dynamics. A classical result related to topologically hyperbolic systems, proved first by Bowen, says that every transitive topologically hyperbolic homeomorphism is a factor of a special subshift (so-called shift of finite type) via a bounded-to-one factor map (e.g. see [6, Theorem 4.3.6]). Then it is not surprising that Theorem 5.2 can be extended to the following.

Theorem 5.4 Let $(X, f)$ be a non-wandering and topologically hyperbolic dynamical system, where $X$ has no isolated points. Then for any nonempty open set $U \subset X$ there exist $m \in \mathbb{N}$ and a subsystem $\left(Y, f^{m}\right)$ for $f^{m}$ with $Y \subset U$, and a conjugacy $\pi:\left(Y, f^{m}\right) \rightarrow\left(\{0,1\}^{\mathbb{Z}}, \sigma\right)$.

Proof For the purposes of this proof, by a bi-infinite $\delta$-pseudo-orbit we mean any sequence $\left\{z_{n}\right\}_{n \in \mathbb{Z}}$ satisfying $d\left(f\left(z_{n}\right), z_{n+1}\right)<\delta$ for every $n$, and $\varepsilon$-tracing by a full orbit $\left\{x_{n}\right\}_{n \in \mathbb{Z}}$ means that $d\left(x_{n}, z_{n}\right)<\varepsilon$ for every $n \in \mathbb{Z}$.

First apply spectral decomposition due to Bowen (see [6, Theorem 3.4.4]) obtaining disjoint closed sets $\Lambda_{1}, \ldots, \Lambda_{r}$ and an integer $q>0$ such that $X=\bigcup_{i=1}^{r} \Lambda_{i}$ and $\left.f^{q}\right|_{\Lambda_{i}}$ is topologically mixing for every $i=1,2, \ldots, r$. Since $X$ does not have any isolated points, none of the sets $\Lambda_{i}$ is a singleton, in particular $f$ is sensitive.

Note that by shadowing and compactness there is a tracing function $\phi$ with the property that for every bi-infinite $\phi(\varepsilon)$-pseudo-orbit $\left\{z_{n}\right\}_{n \in \mathbb{Z}}$ there is a full orbit $\left\{x_{n}\right\}_{n \in \mathbb{Z}}$ which $\varepsilon$-traces it, that is $d\left(x_{n}, z_{n}\right)<\varepsilon$ for every $n \in \mathbb{Z}$. Fix an expansive constant $\beta>0$ and observe that if $\varepsilon<\beta / 2$ then the tracing full orbit above is uniquely determined.

By Theorem 4.2, there is a minimal sensitive $\operatorname{subsystem}(M, f)$ with $M \cap U \neq \emptyset$. Let $u \in M \cap U$, and let $\lambda>0$ be a sensitivity constant for $(M, f)$ such that $\bar{B}(u, \lambda) \subset U$. Let $S:=N_{f}\left(u, B\left(u, 4^{-1} \lambda\right)\right)$. Write $S=\left\{0=s_{0}<s_{1}<s_{2}<\cdots\right\}$, and let $q \in \mathbb{N}$ be a bound for the gaps in the syndetic set $S$. Choose $0<\varepsilon<\beta / 3$ with the property that $d(a, b) \leq 4 \varepsilon$ implies $d\left(f^{i}(a), f^{i}(b)\right)<2^{-1} \lambda$ for every $a, b \in X$ and $0 \leq i \leq q$. Keep in mind that $\varepsilon<8^{-1} \lambda$ by our choice.

Now proceed exactly as in the proof of Theorem 5.1 by choosing $C(0), C(1)$, etc. till defining the two finite sequences in (5.1) and (5.2):

$$
\eta(0)=\left(u, f(u), \ldots, f^{m-1}(u)\right), \quad \text { and } \eta(1)=\left(v, f(v), \ldots, f^{m-1}(v)\right) .
$$


For any point $x \in\{0,1\}^{\mathbb{Z}}$ let $z_{x}=q_{0}$, where $\left\{q_{n}\right\}_{n \in \mathbb{Z}}$ is the full orbit which is $\varepsilon$-tracing the bi-infinite $\phi(\varepsilon)$-pseudo orbit

$$
\xi_{x}=\left(\ldots y_{-1} y_{0} y_{1} y_{2} \ldots\right)=\left(\ldots \eta\left(x_{-1}\right) . \eta\left(x_{0}\right) \eta\left(x_{1}\right) \eta\left(x_{2}\right) \eta\left(x_{3}\right) \ldots\right)
$$

where symbol "." in the above sequence indicates the position of $y_{0}$, i.e. $y_{0}, y_{1}, \ldots$ are elements just after ".".

Note that the point $z_{x}$ is unique by the choice of $\varepsilon$. Moreover, it may be seen that $z_{x} \neq z_{x^{\prime}}$ if $x \neq x^{\prime}$ since $d\left(c_{0}, c_{1}\right) \geq 2 \varepsilon$ for any $\left(c_{0}, c_{1}\right) \in C(0) \times C(1)$. Hence setting $Y=\left\{z_{x}: x \in\{0,1\}^{\mathbb{Z}}\right\} \subset C(0) \cup C(1) \subset U$, we see that the map $\pi: Y \rightarrow\{0,1\}^{\mathbb{Z}}$ defined as $\pi\left(z_{x}\right)=x$ is a well-defined bijection. Clearly, $\pi \circ f^{m}=\sigma \circ \pi$, and it may be verified as in the proof of Theorem 5.1 that $\pi$ is continuous. This completes the proof.

Example 5.5 Take any continuous map $f:[0,1] \rightarrow[0,1]$ and assume that $([0,1], f)$ is a non-wandering dynamical system with shadowing. A particular example here is the full tent map $f(x)=1-|1-2 x|$ or various other maps from the family of tent maps [15].

It is easy to verify that $f$ is mixing (it is straightforward to show that $N(U, V)$ is cofinite for any two open intervals $U, V)$. Now, if we fix any open interval $U$, then shadowing with mixing immediately imply that there are two disjoint closed intervals $J_{0}, J_{1} \subset U$ and $m>0$ such that $f^{m}\left(J_{0}\right) \cap f^{m}\left(J_{1}\right) \supset J_{0} \cup J_{1}$. But then, by results of [24] (see the proof of [24, Theorem 9]), there is a closed set $\Lambda \subset J_{0} \cup J_{1} \subset U$ and $s>0$ such that $\Lambda$ is invariant for $f^{m+s}$ such that $\left(\Lambda, f^{m+s}\right)$ is conjugated to $\left(\{0,1\}^{\mathbb{N}}, \sigma\right)$.

We have seen that for an infinite non-wandering system with shadowing, cexpansivity is a sufficient condition to make the factor map in Theorem 5.2 a conjugacy. The examples of interval maps given above are not c-expansive (see [6]), and this shows that c-expansivity is not a necessary condition. So the following question is natural.

Question 1 Is there a simple necessary and sufficient condition for the factor map in Theorem 5.2 to be a conjugacy?

Remark 5.6 We proved so far that every non-wandering and sensitive dynamical system having shadowing contains dense collections of two types of minimal systems:

(1) sensitive, almost 1-1 extensions of odometers,

(2) systems with positive entropy and without regularly recurrent points (this is much stronger than simply saying that $M(f) \backslash R R(f)$ is dense).

It is well known that for non-wandering topologically hyperbolic dynamical system there is $m>0$ and there are disjoint closed sets $D_{1}, \ldots, D_{k}$ invariant for $f^{m}$ such that $\left(D_{i}, f^{m}\right)$ is mixing for each $i=1,2, \ldots, k$ (it is the so-called spectral decomposition due to Smale, e.g. see [6]). It can also be proved that each $\left(D_{i}, f^{m}\right)$ contains plenty of minimal weakly mixing subsystems (because it is bounded-to-one factor of a shift of finite type [6]). This is no longer true if we drop expansivity assumption. 
Example 5.7 Let $(X, f)$ be a dynamical system obtained as the Cartesian product of an odometer and full shift. Then $(X, f)$ is transitive, and has shadowing since both the odometer and the full shift have the shadowing property. But if $M \subset X$ is a minimal set for $f^{m}$ then $\left(M, f^{m}\right)$ has an odometer as its factor (it is enough to take projection onto the first co-ordinate), in particular its maximal equicontinuous factor is not a singleton. It implies that $\left(M, f^{m}\right)$ is not weakly mixing.

The above observations motivate the following question.

Question 2 Does every weakly mixing dynamical system with shadowing contain a weakly mixing minimal subsystem?

Motivated by Corollary 3.3 we may also ask another question about the possible existence of minimal subsystems with highly non-chaotic behavior:

Question 3 Does every non-wandering sensitive dynamical system with shadowing contain an equicontinuous minimal subsystem?

\section{Answering two questions: about semi-distality and entropy points}

We answer two more questions from [25]. First we show that an infinite transitive semi-distal system with shadowing must be a minimal equicontinuous system on a Cantor space, completing the results of [25, Theorem 5].

We recall two interesting known facts.

Theorem 6.1 (Theorem 2.7(c), [4]) If $(X, f)$ is transitive and semi-distal, then $(X, f)$ is minimal.

Theorem 6.2 (Proposition 2.7, [32]) Let $(X, f)$ be a dynamical system, let $x \in R(f)$ and let $A$ be a minimal set contained in $\overline{O(f, x)}$. Then there is $y \in A$ such that $(x, y) \in \operatorname{Prox}(f) \cap R(f \times f)$.

Now we have two (related) proofs for our promised result.

Corollary 6.3 Let $(X, f)$ be an infinite transitive dynamical system having shadowing. If $f$ is semi-distal, then $f$ is a minimal equicontinuous map of the Cantor space.

Proof It suffices to show that $f$ is minimal, for then the required conclusion follows by Theorem 6 of [25]. The minimality of $f$ follows either directly by Theorem 6.1, or in the following manner: if $f$ is not minimal, then by considering a transitive point $x$ and applying Theorem 6.2, we see that there exists $(x, y) \in R(f \times f) \cap \operatorname{Prox}(f) \backslash \Delta$, and in particular $(x, y) \notin A s y(f)$ also, which contradicts the assumption that $f$ is semi-distal.

We digress a little bit to note down a Corollary of Theorem 6.2.

Corollary 6.4 Let $(X, f)$ be a dynamical system and $x, y \in X$. If $(x, y) \in \operatorname{Prox}(f)$ then there is $z \in M(f) \cap \overline{O(f, x)} \cap \overline{O(f, y)}$ such that $((x, y),(z, z)) \in \operatorname{Prox}(f \times f)$. In addition, if $(x, y) \in R(f \times f)$, then the point $z$ can be chosen to satisfy the extra property that $(x, y, z) \in R(f \times f \times f)$. 
Proof Let $g=f \times f, \Delta_{X}$ be the diagonal in $X^{2}$ and $A=\Delta_{X} \cap \overline{O((x, y), g)}$. Then $A$ is closed, $g$-invariant, and $A \neq \emptyset$ since $(x, y) \in \operatorname{Prox}(f)$. Applying [18, Proposition 8.6] we get $(z, z) \in A \cap M(g)$ such that $((x, y),(z, z)) \in \operatorname{Prox}(g)$. If we also have $(x, y) \in R(g)$, then by Theorem 6.2 we may additionally assume that $z$ satisfies the property $(x, y, z, z) \in R(g \times g)$.

It was proved in [25] that if $f$ has shadowing then sensitive points are entropy points, and hence when $f$ is a sensitive dynamical system with shadowing then $E_{p}(f)=X$. Since $f\left(E_{p}(f)\right) \subset E_{p}(f)$ and $E_{p}(f)$ is closed, we immediately obtain that $E_{p}(f)=$ $X$ in any minimal system with positive topological entropy (and clearly, every such a system is sensitive). This motivated the author of [25] to state the following question:

- If $X$ is a compact metric space and $f: X \rightarrow X$ is a transitive map with $h(f)>0$, is it true that $E_{p}(f)=X$ ?

The following theorem shows that the answer for the above question from [25] is negative.

Theorem 6.5 There is a mixing subshift $(X, \sigma)$ with positive entropy over the alphabet $\{a, b, c\}$ such that

(i) X contains elements starting with a, but

(ii) $X$ has no entropy point starting with $a$.

Proof Let $X \subset\{a, b, c\}^{\mathbb{N}}$ be the collection of all $x$ satisfying the following condition: if $w$ is a word of length $2^{n}$ (where $n \in \mathbb{N}$ ) appearing in $x$ and if $w$ starts with the letter $a$, then $\#\left\{i: w_{i} \neq b\right\} \leq n$. It is easy to see $(X, \sigma)$ is a subshift. Since $\{b, c\}^{\mathbb{N}} \subset X$, the system $(X, \sigma)$ has positive entropy.

Let $L(X)$ denote the language of $X$. If $u, w \in L(X)$, then $u b^{k} w b^{\infty} \in X$ for all sufficiently large $k$, and this shows $(X, \sigma)$ is mixing. Since $a b^{\infty} \in X$, condition (i) is also satisfied.

Let $\alpha(m)$ be the number of words $w \in L(X)$ of length $m$ and starting with $a$. Consider $w \in L(X)$ of length $2^{n}$ such that $w$ starts with $a$. Suppose \#\{i: $\left.w_{i} \neq b\right\}=k$. The number of ways to choose $k$ positions from $2^{n}$ positions is bounded from the above by $\left(2^{n}\right)^{k}=2^{k n}$. The number of ways to fill $k$ positions with two letters is $2^{k}$. Hence

$$
\alpha\left(2^{n}\right) \leq \sum_{k=0}^{n} 2^{k n+k} \leq(n+1) 2^{n^{2}+n} \leq\left(2^{2 n^{2}}\right)^{2 n}=2^{4 n^{3}}
$$

for all $n \in \mathbb{N}$. Now, given $m \in \mathbb{N}$, take $n \in \mathbb{N}$ such that $2^{n-1} \leq m<2^{n}$. Then $\alpha(m) \leq \alpha\left(2^{n}\right)$, and therefore

$$
\frac{\log \alpha(m)}{m} \leq \frac{\log \alpha\left(2^{n}\right)}{2^{n-1}} \leq \frac{4 n^{3} \log 2}{2^{n-1}}
$$

This implies that

$$
h\left(C_{X}(a),\left.\sigma\right|_{X}\right)=\limsup _{m \rightarrow \infty} \frac{\alpha(m)}{m} \leq \lim _{n \rightarrow \infty} \frac{8 n^{3} \log 2}{2^{n}}=0 .
$$


where $C_{X}(a)=\left\{x \in X: x_{0}=a\right\}$. This gives (ii), completing the proof.

It is known that on many manifolds, shadowing homeomorphisms with positive entropy are generic [21]. Hence the following question is natural to ask:

Question 4 Is there a compact topological manifold $M$ and a transitive homeomorphism $f: M \rightarrow M$ such that $\emptyset \neq E_{p}(f) \neq M$ ?

Acknowledgment We thank the referees for their valuable comments and suggestions to improve the paper. Research of Piotr Oprocha was supported by Narodowe Centrum Nauki (National Science Center) in Poland, grant no. DEC-2011/03/B/ST1/00790.

Open Access This article is distributed under the terms of the Creative Commons Attribution License which permits any use, distribution, and reproduction in any medium, provided the original author(s) and the source are credited.

\section{References}

1. Akin, E.: On chain continuity. Discret. Cont. Dynam. Sys. 2, 111-120 (1996)

2. Akin, E.: Recurrence in topological dynamics. Furstenberg families and Ellis actions, the university series in mathematics. Plenum Press, New York (1997)

3. Akin, E., Auslander, J., Berg, K.: When is a transitive map chaotic? Convergence in ergodic theory and probability (Columbus, OH, 1993), vol. 5, pp. 25-40. Ohio State University of Mathematics Research Institute Publications., de Gruyter (1996)

4. Akin, E., Auslander, J., Glasner, E.: The topological dynamics of Ellis actions. Mem. Amer. Math. Soc. 195, 913 (2008)

5. Anosov, D.V.: On a class of invariant sets for smooth dynamical systems. In: Proceeding fifth international conference on nonlinear oscillations, vol. 2, pp. 39-45. Mathematics Institute Ukrainian Academic Science, Kiev (in Russian) (1970)

6. Aoki, N., Hiraide, K.: Topological theory of dynamical systems. North-Holland (1994)

7. Balibrea, F., Downarowicz, T., Hric, R., Snoha, L.: Almost totally disconnected minimal systems, ergodic theory dynam. Systems 29, 737-766 (2009)

8. Bernardes, N., Darji, U.: Graph theoretic structure of maps of the Cantor space. Adv. Math. 231, $1655-1680(2012)$

9. Bonatti, C., Díaz, L., Turcat, G.: Pas de "shadowing lemma" pour des dynamiques partiellement hyperboliques, (French) [there is no shadowing lemma for partially hyperbolic dynamics]. C. R. Acad. Sci. Paris 330, 587-592 (2000)

10. Blanchard, F., Glasner,.E., Kolyada, S., Maass, A.: On Li-Yorke pairs. J. fur die reine und angewandte Mathematik (Crelle's J.) 547, 51-68 (2002)

11. Blanchard, F., Host, B., Ruette, S.: Asymptotic pairs in positive-entropy systems. Ergodic Theory Dynam. Syst. 22(3), 671-686 (2002)

12. Blanchard, F., Kwiatkowski, J.: Minimal self-joinings and positive topological entropy II. Studia Math. 128, 121-133 (1998)

13. Block, L.S., Coppel, W.A.: Dynamics in one dimension, Lecture Notes in Mathematics, vol. 1513. Springer, Berlin (1992)

14. Bowen, R.: Equilibrium states and the ergodic theory of Anosov diffeomorphisms. In: Lecture notes in mathematics, vol. 470. Springer-Verlag, Berlin-New York (1975)

15. Coven, E.M., Kan, I., Yorke, J.A.: Pseudo-orbit shadowing in the family of tent maps. Trans. Amer. Math. Soc. 308(1), 227-241 (1988)

16. Downarowicz, T.: Survey of odometers and Toeplitz flows, algebraic and topological dynamics, vol. 385, pp. 7-37. Contemporary Mathematics-American Mathematics Society, Providence (2005)

17. Downarowicz, T., Ye, X.: When every point is either transitive or periodic. Colloq. Math. 93(1), 137$150(2002)$

18. Furstenberg, H.: Recurrence in ergodic theory and combinatorial number theory. Princeton University Press, Princeton (1981) 
19. Huang, W., Li, H., Ye, X.: Family-independence for topological and measurable dynamics. Trans. Amer. Math. Soc. 364, 5209-5242 (2012)

20. Huang, W., Ye, X.: Dynamical systems disjoint from any minimal system. Trans. Amer. Math. Soc. 357, 669-694 (2005)

21. Kocielniak, P., Mazur, M.: Chaos and the shadowing property. Topol. Appl. 154, 2553-2557 (2007)

22. Li, J.: Transitive points via Furstenberg family. Topol. Appl. 158, 2221-2231 (2011)

23. Mai, J.H., Ye, X.: The structure of pointwise recurrent maps having the pseudo orbit tracing property. Nagoya Math. J. 166, 83-92 (2002)

24. Moothathu, T.K.S.: Syndetically proximal pairs. J. Math. Anal. Appl. 379, 656-663 (2011)

25. Moothathu, T.K.S.: Implications of pseudo-orbit tracing property for continuous maps on compacta. Top. Appl. 158, 2232-2239 (2011)

26. Ombach, J.: Equivalent conditions for hyperbolic coordinates. Topol. Appl. 23, 87-90 (1986)

27. Oprocha, P.: Spectral decomposition theorem for non-hyperbolic maps. Dyn. Syst. 23, 299-307 (2008)

28. Palmer, K.: Shadowing in dynamical systems. Theory and applications, mathematics and its applications, vol. 501. Kluwer Academic Publishers, Dordrecht (2000)

29. Pilyugin, S.Y.: Shadowing in dynamical systems, Lecture Notes in Mathematics, vol. 1706. SpringerVerlag, Berlin (1999)

30. Pilyugin, S.Y., Tikhomirov, S.: Lipschitz shadowing implies structural stability. Nonlinearity 23(10), 2509-2515 (2010)

31. Sakai, K.: Pseudo orbit tracing property and strong transversality of diffeomorphisms on closed manifolds. Osaka J. Math. 31, 373-386 (1994)

32. Shao, S.: Proximity and distality via Furstenberg families. Topol. Appl. 153, 2055-2072 (2006)

33. Ye, X., Zhang, G.: Entropy points and applications. Trans. Amer. Math. Soc. 359(12), 6167-6186 (2007) 